\title{
LA VIVIENDA DEL COMPACTO (TECNOLOGÍA DE LAS INSTALACIONES EN LA VIVIENDA)
}

\author{
(THE COMPACT HOUSING - HOUSING CONSTRUCTION TECHNOLOGY-)
}

\section{José Miguel Reyes, Arquitecto.}

E. T. Bretón de los Herreros, 10. Madrid - España.

\section{RESUMEN}

Se presenta en este articulo una llamada de atención sobre el reciente fenómeno del COMPACTO, como objeto de diseño industrial dentro del mercado de consumo de la tecnología de las instalaciones domésticas. Se trata de señalar y analizar algunas de sus características culturales y técnicas más destacadas:

- productivas: industria, serie;

- de consumo: mercado de componentes;

- espaciales: unidades separables/contenedores: flexibilidad.

A partir de las mismas se revisa la trayectoria conceptual de estos productos, y de sus influencias espaciales, a través de una serie de destacados ejemplos de arquitectura: desde "La Casa Dominó", de Le Corbusier, hasta "La Robot-House", de R. Herron. Se cuestiona su función dentro de la propia cultura que los crea. Por último, ante su cierta presencia, se describen cuatro recientes ejemplos de COMPACTO.

\section{SUMMARY}

This article calls to attention the recent COMPACT phenonemum, as the object of industrial design in the consumer world of technology in domestic installations. Its aim is to point out and analyze some of its most outstanding cultural and technical characteristics:

- production: industry, series:

- consumer: components' market;

- spatial: separable units/containers: flexibility.

With these characteristics as starting points the conceptual development of these products is reviewed along with its spatial influences by means of a series of outstanding examples of architecture: from "/a Casa Dominó" - Le Corbusier, to "la Robot-Hause" . R. Herron. Its function within its own cultural sphere which creates these products is discussed. Finally, in the face of the considerable presence of the COMPACT, four recent examples are described.

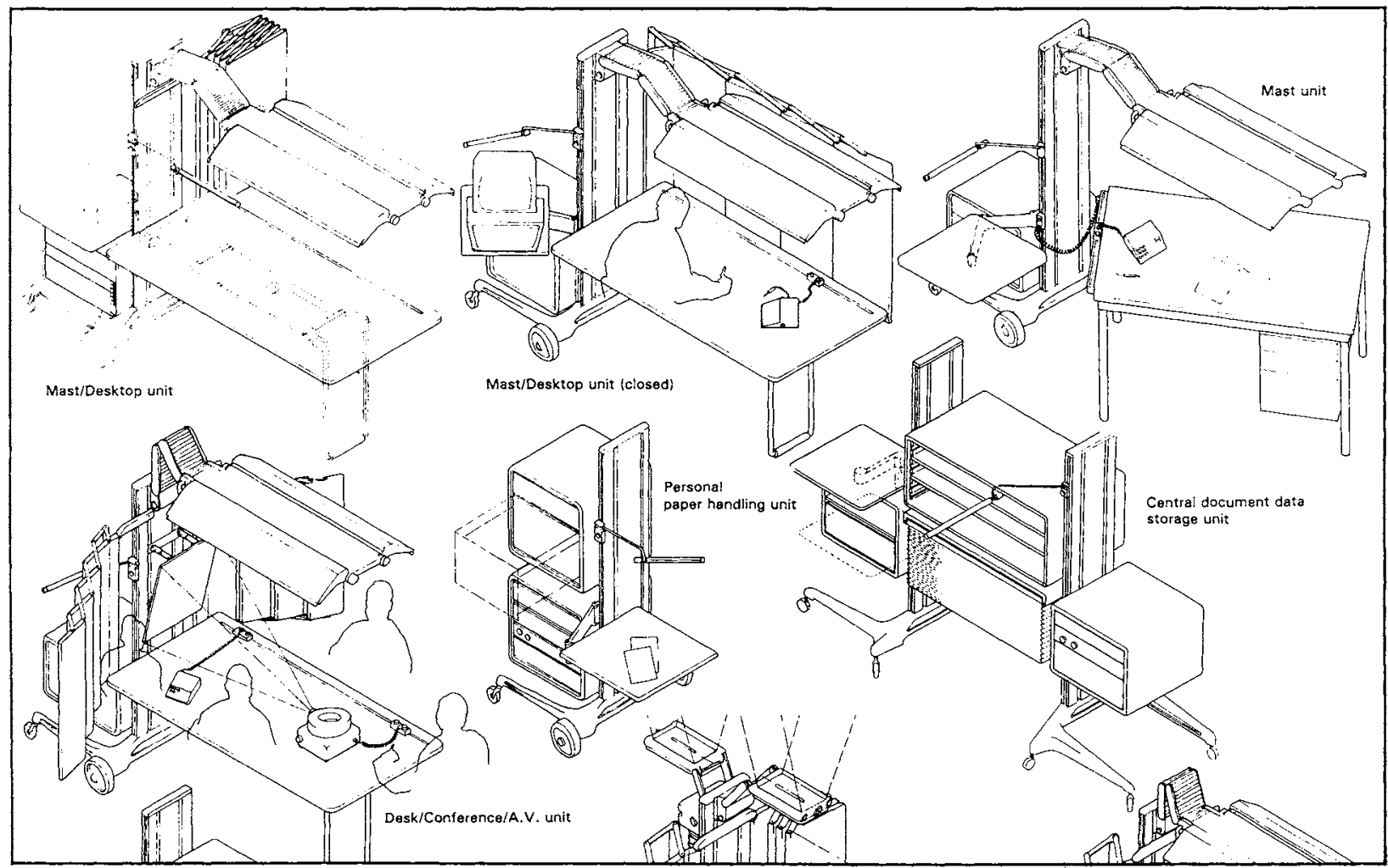



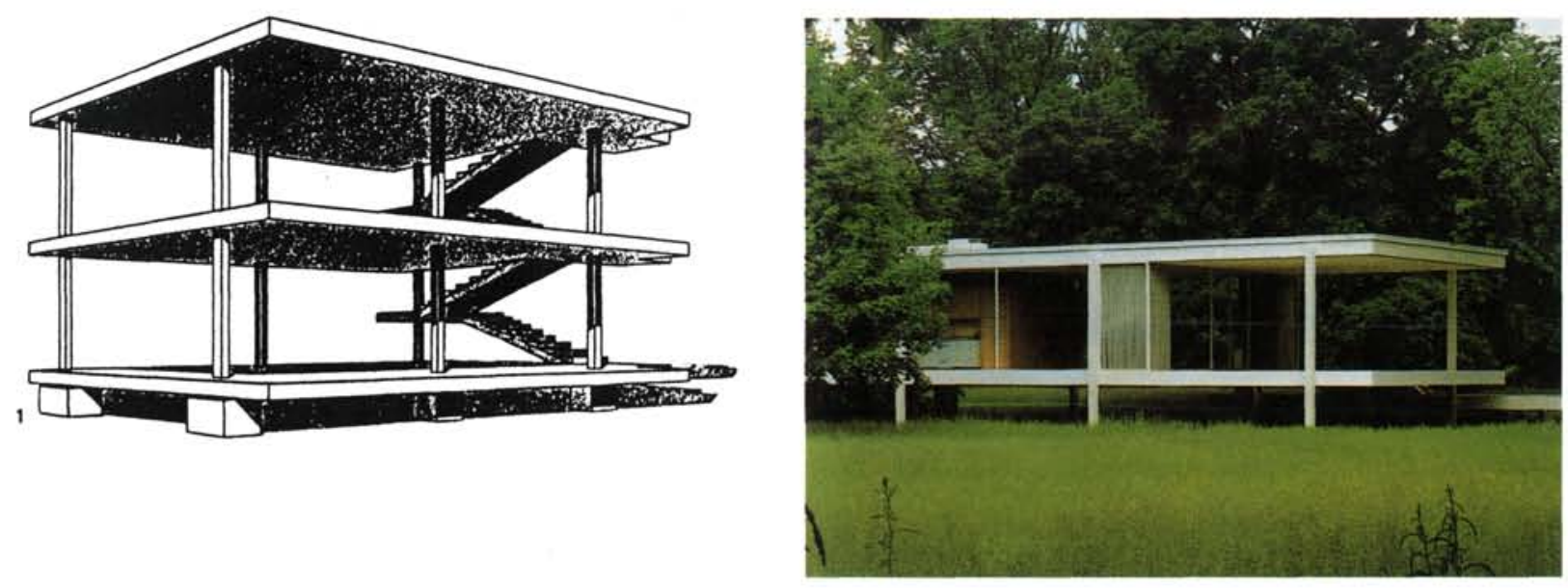

\section{PILOTIS}

\section{Dominó EL CONCEPTO}

- planta libre/altura constante,

- se rompen esquemas convencionales,

- "libre" distribución en planta,

- fabricación in situ/solución fija.
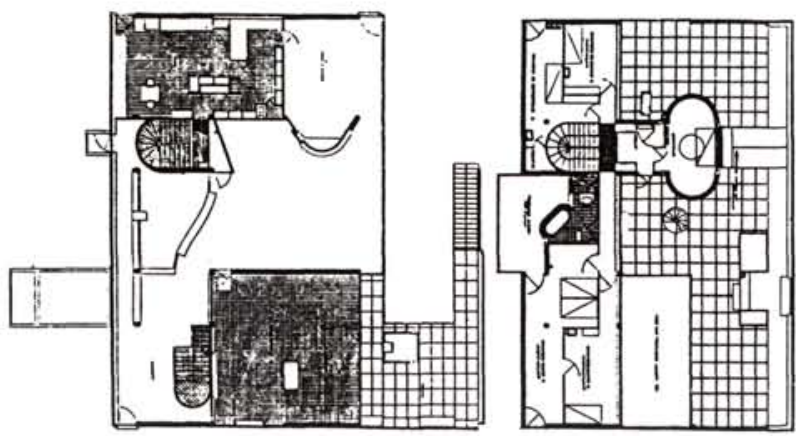

(C) Consejo Superior de Investigaciones Científicas Licencia Creative Commons 3.0 España (by-nc)

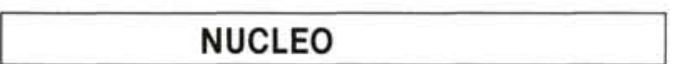

\section{PILOTIS}

1945 Fansworth EL MODELO

- Ia tecnología fija la solución:

núcleo y perimetro determinan la distribución,

- fabricación industrial de las piezas.

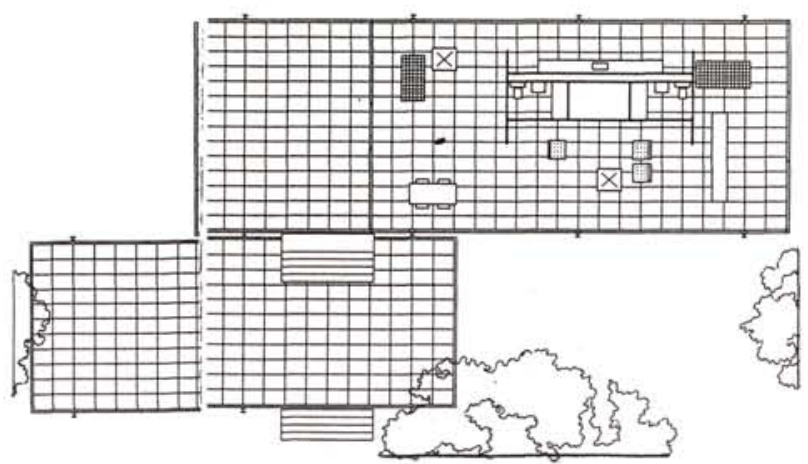

http://informesdelaconstruccion.revistas.csic.es 


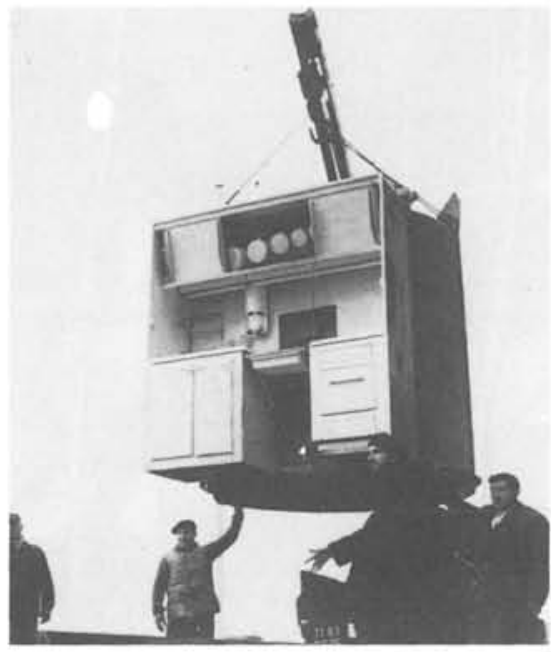

MONOBLOCK

\begin{tabular}{|l|}
\hline NUCLEO \\
\hline PILOTIS \\
\hline
\end{tabular}

1955 J. Prouve EL PROTOTIPO

- fabricación industrial de piezas y bloques.

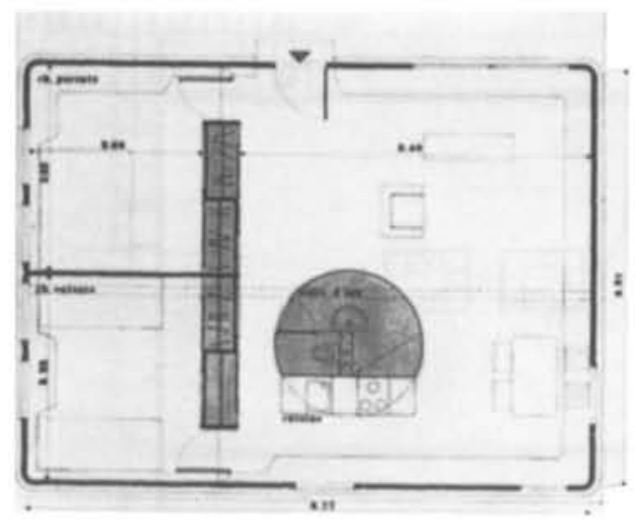

Dentro del actual campo del diseño industrial están proliferando, cada vez con más frecuencia, toda una gama de objetos de consumo doméstico que, comercialmente, vienen a denominarse COMPACTOS:

- ¿qué son?

- ¿qué caracteristicas técnicas aportan?

- ¿a qué necesidades responden?

- ¿cuáles son sus antecedentes?

- ¿cuáles serian sus posibles influencias sobre el mo. do de vida y la habitación?

Éstas son solamente algunas de las muchas cuestiones que el tema sugiere. El hecho de que el fenómeno se encuentre en incipiente desarrollo hace que, por una parte, sea más dificil su análisis; pero, a cambio, también lo convierte en un tema aún más interesante.

En cuanto a su definición se podrian señalar algunas de sus caracteristicas funcionales, formales, y de producción. Se nos presentan como aparatos que, con una apariencia de mecanismo unitario "monoblock", ofrecen al usuario una variedad multifuncional de aplicaciones integradas.

Estos aparatos suelen estar asociados a la tecnologia de las instalaciones ofreciendo los servicios propios de la vivienda. Partiendo de los electrodomésticos convencionales (lavadora, frigorifico, horno, TV., radio,...) se agrupan según ciertas especializaciones:

- Comunicación: trabajo (mesas informatizadas); descanso (audiovisuales, iluminación); ocio.

- Almacenamiento: alimentación (frigoríficos); objetos (ficheros).

- Aseo y limpieza: cápsulas sanitarias; instalaciones monoblock; lavadoras.

- Calefacción y refrigeración: fancoils, extractores...

Esta agrupación de funciones permite su fabricación industrial como objetos monoblock, que recogen la tradición y las ventajas de la serie en: la náutica, la aeronáutica, el ferrocarril o el automovilismo para esta clase de funciones (como puede apreciarse toda la enumeración corresponde al sector transportes). Este aspecto unitario que parte del monocasco, como modelo industrial, tiene sus ventajas e inconvenientes. 


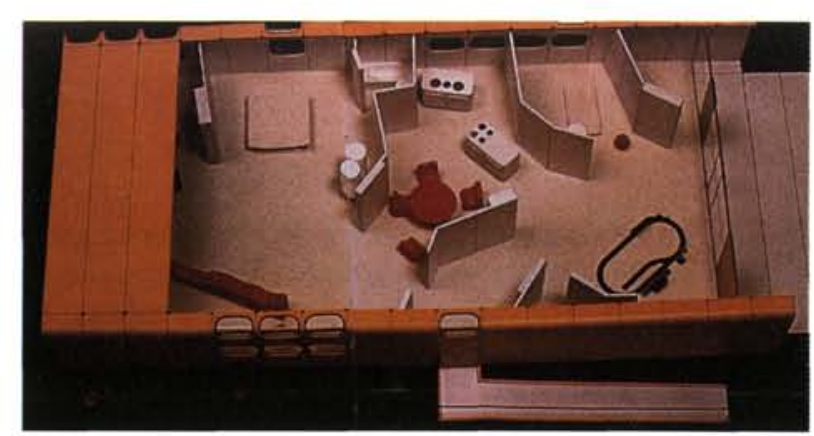

\section{MOVIL}

\begin{tabular}{|l|}
\hline MONOBLOCK \\
\hline NUCLEO \\
\hline PILOTIS \\
\hline
\end{tabular}

1968 Zip-up EL MANIFIESTO

- producción industrial,

- distribución libre: movilidad total de piezas y bloques de instalación.

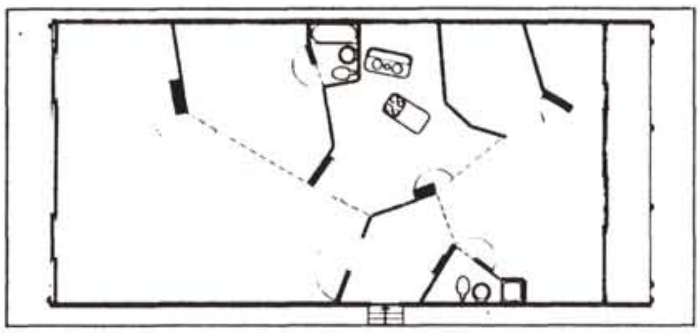

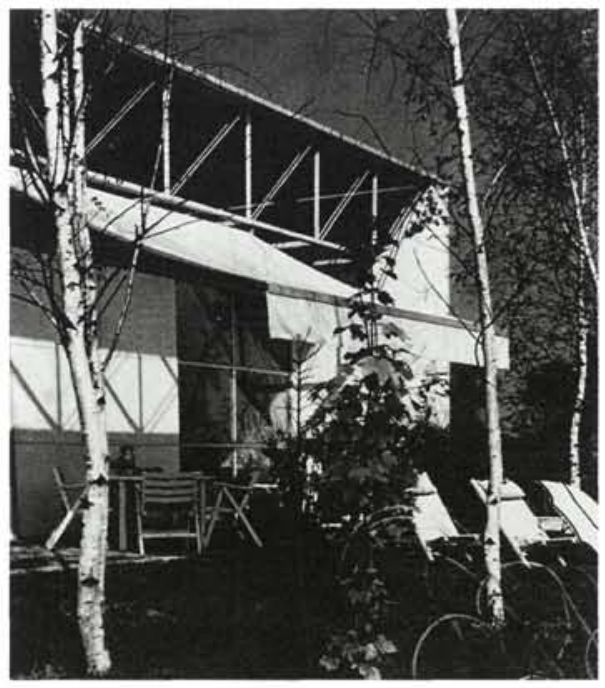

\section{PIEL}

\begin{tabular}{|l|}
\hline MOVIL \\
\hline MONOBLOCK \\
\hline NUCLEO \\
\hline PILOTIS \\
\hline
\end{tabular}

1972 R. Piano EL ENSAYO

- producción y mercado industrial,

- tratamiento del perimetro (orientación): "situación del núcleo: fija,

- una zonificación que posibilita ciertas distribu. ciones.

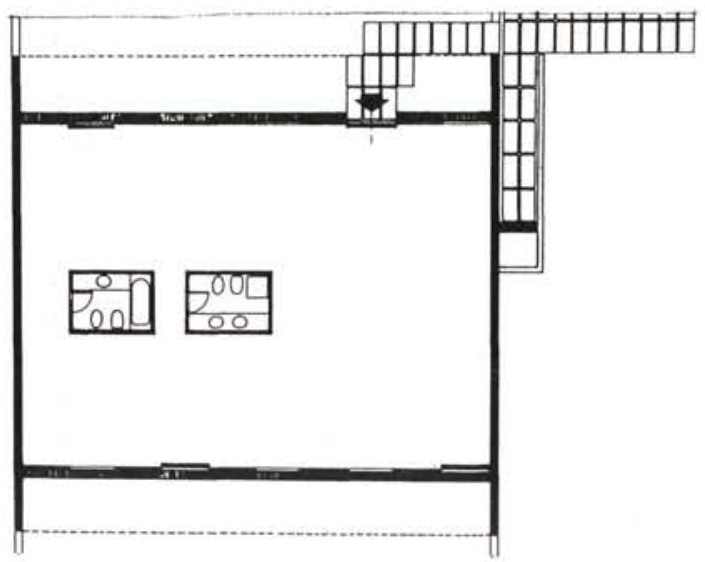

http://informesdelaconstruccion.revistas.csic.es 

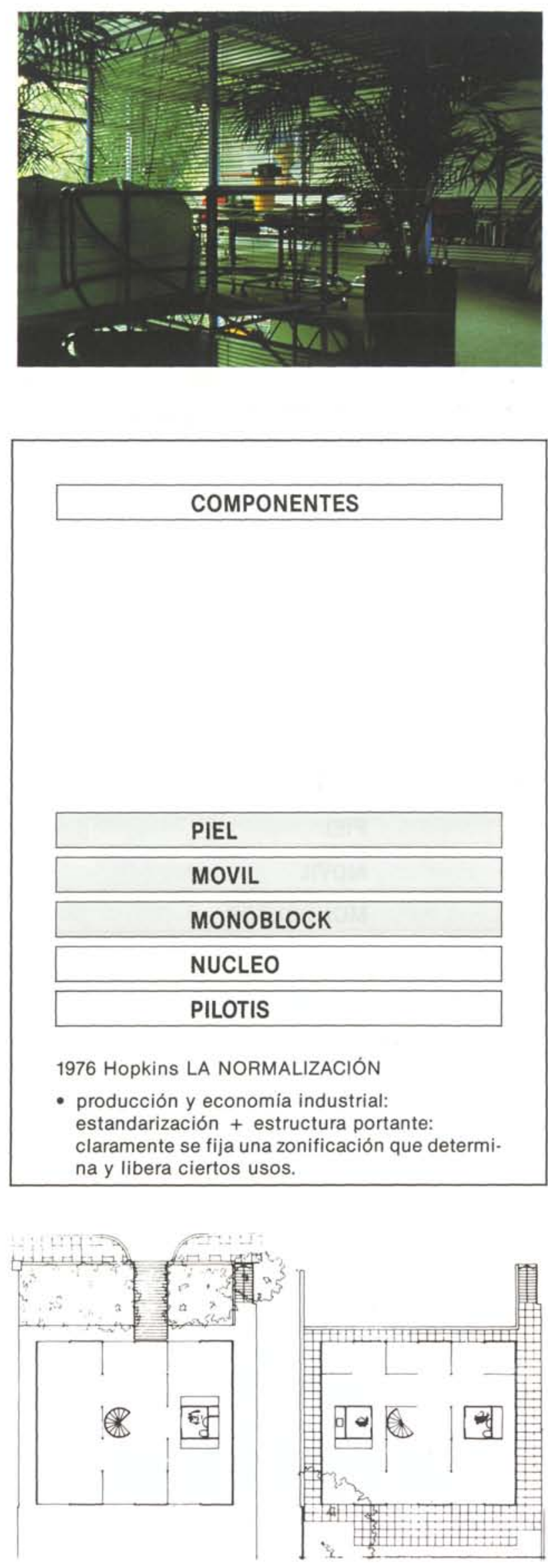

(c) Consejo Superior de Investigaciones Científicas Licencia Creative Commons 3.0 España (by-nc)
Cabe señalar, como una de sus importantes ventajas, su fácil desplazamiento, por lo cual en su forma de manejo se acerca al concepto de mueble. En su movimiento se desplaza no solamente cierta función individual, como en el caso del electrodoméstico tradicional, sino todo un grupo de ellas que caracterizan y singularizan el espacio en que el COMPACTO se sitúa.

Sin embargo, ese mismo carácter unitario aparece como un inconveniente importante a la hora de su reparación parcial, empujándonos a adquirir otro "nuevo aparato" si el fabricante "no ha previsto" la fácil sustitución de sus elementos o piezas. Este inconveniente es superable cuando tales "compactos" se nos ofrecen atendiendo al concepto de sistema integrado. Tales sistemas responden a la idea de producción industrial a partir de componentes compatibles.

Nos encontramos asi ante "compactos" integrados por componentes compatibles que, en su compatibilidad, pueden añadir, sustituir o quitar componentes, variando sus prestaciones. Tanto en el caso de su movilidad, como en el de su función, nos encontramos ante una importante propiedad de los mismos: flexibilidad.

La misma influye, obviamente, sobre los sistemas de construcción, la habitación, la forma de vida,...

En general, el concepto está asociado a la tecnologia de las instalaciones, y la tecnología de las instalaciones ha hecho evolucionar muy fuertemente a la vivienda urbana desde el siglo pasado: luz, agua,...

En el momento en que estas tecnologias se incorporan a la vivienda, comienzan a surgir cambios importantes en la misma. Por una parte se plantea una especialización de sus espacios, pero por otra, también se discuten la localización e importancia de las instalaciones en su definición: comienza la trayectoria de "LA MÁQUINA DE VIVIR". De una parte, la vivienda mínima conduce a una estandarización y automatización del diseño cada vez más inerte. De otra, la evolución de la tecnologia de las instalaciones domésticas influye de manera constante en la evolución de la vivienda.

1914, CASA DOMINÓ: dos planos horizontales. Planta libre: altura constante. Rotura de todos los esquemas previos. No hay pautas. La colocación de las instalaciones fija la solución de la vivienda.

1986, ROBOT HOUSE: dos planos horizontales. Planta libre: altura variable. Discusión de esquemas previos. Se insinuan ciertas pautas. La colocación de las ins. talaciones no fija la solución de la vivienda. 

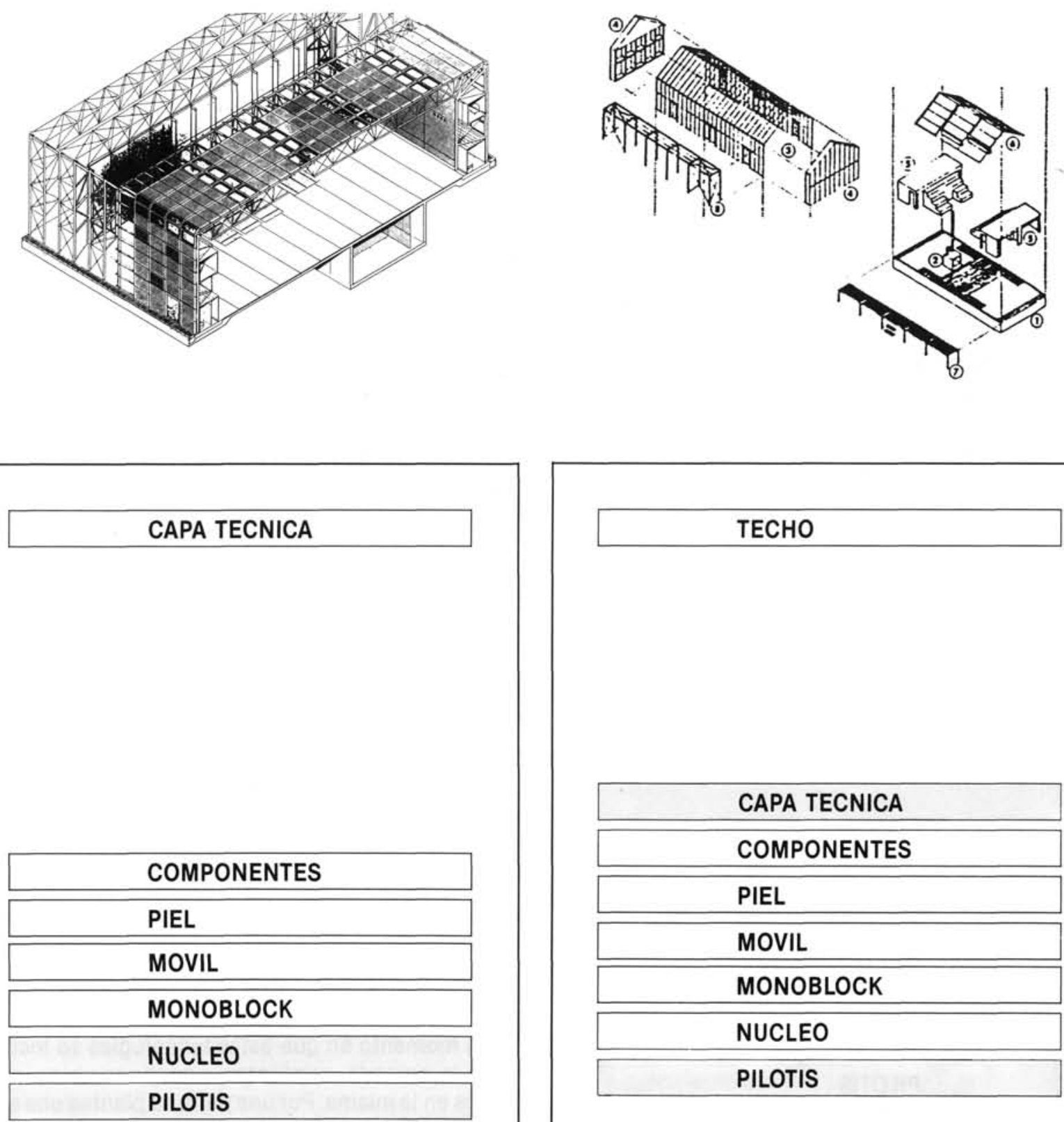

1981 Sainsbury LA TECNOLOGIA

- el orden y tratamiento del perímetro exterior, libera la situación del mobiliario técnico interior.

\begin{tabular}{|l|}
\hline CAPA TECNICA \\
\hline COMPONENTES \\
\hline PIEL \\
\hline MOVIL \\
\hline MONOBLOCK \\
\hline NUCLEO \\
\hline PILOTIS \\
\hline
\end{tabular}

1985 Serre LA NAVE

- doble altura: posibilidades y valoraciones espa. ciales distintas: mayor especialización para unos usos e indeterminación para otros.
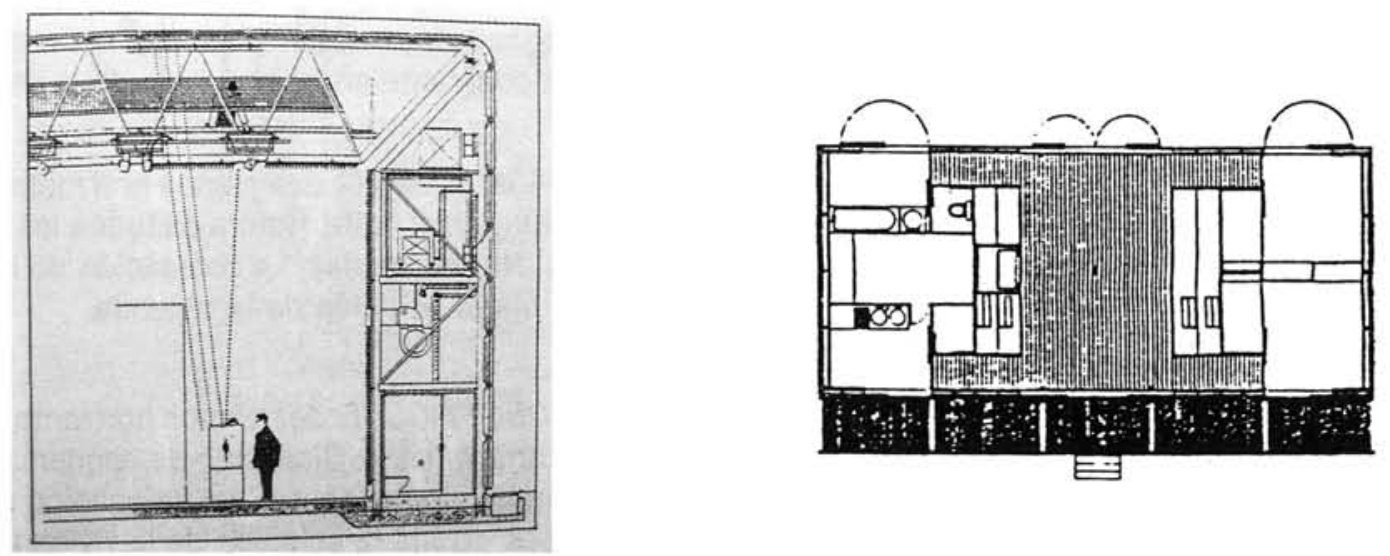


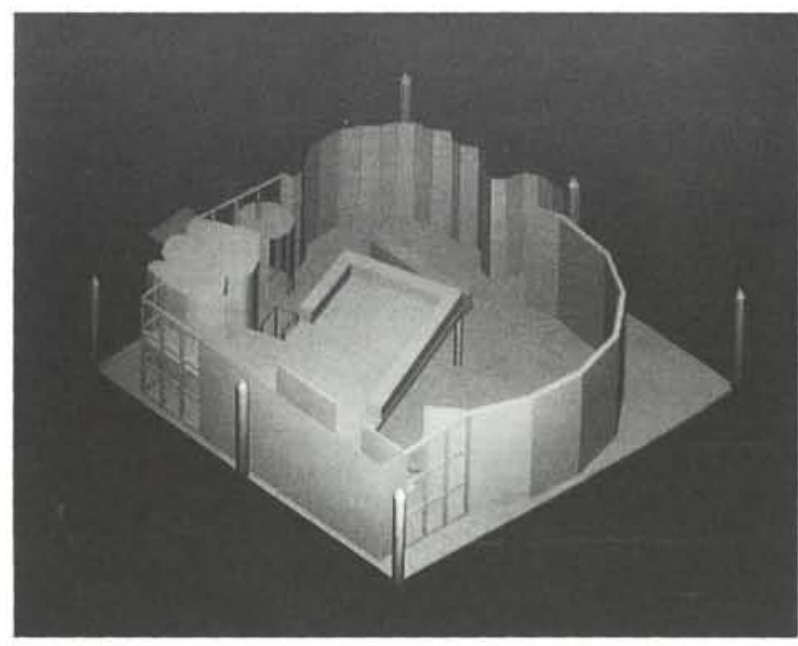

COMPACTO

\begin{tabular}{|l|}
\hline TECHO \\
\hline CAPA TECNICA \\
\hline COMPONENTES \\
\hline PIEL \\
\hline MOVIL \\
\hline MONOBLOCK \\
\hline NUCLEO \\
\hline PILOTIS \\
\hline
\end{tabular}

1986 Robot House LA CIBERNETICA

- control electrónico del medio entorno a una diversidad de mobiliario compacto que sirve y es servido.

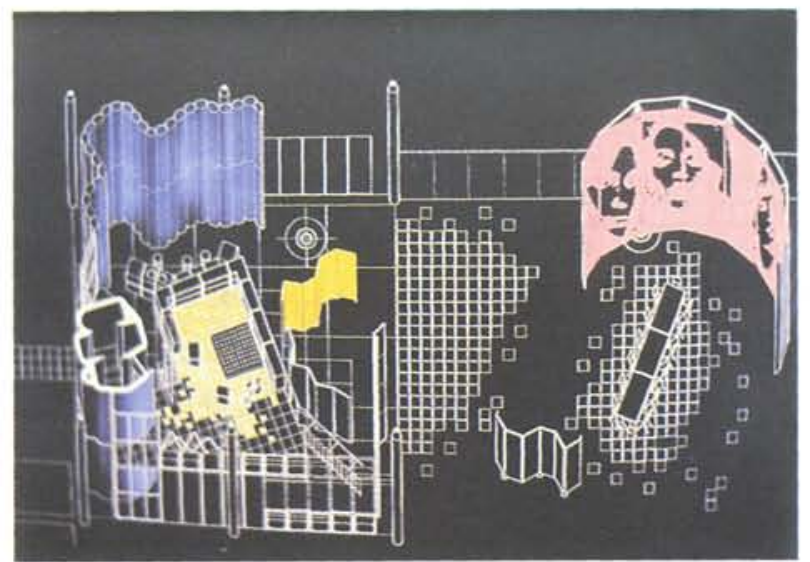

¿A qué responde esta evolución?

Que los modos de vivir y de la vivienda siempre están cambiando, es innegable (tanto en medio rural como urbano). Ahora bien ¿en qué sentido se produce es. te cambio? Es una respuesta siempre dificil de contestar, o que quizás sea mejor no tener que contestar nunca, dejando asi siempre la puerta abierta a la evolución y experimentación de la sociedad. Pero, actualmente ¿en dónde se notan más tales cambios, dada la velocidad a que se producen?, ¿qué elementos de la edifica. ción fijan su estabilidad y cuáles pueden favorecer su metamorfosis?

Si seguimos la trayectoria de esta evolución desde la Casa Dominó hasta la Robot House, vemos cómo para esta clase de construcción industrial, dependiente de sus tecnologias y servicios, cada vez se depura más el concepto de soporte-unidad separable (S.A.R.).

Soporte que viene definido por las condiciones del lu. gar y el tipo de agrupación: acceso, orientación, vistas, recursos, cultura,... y unidades separables: cerramiento, muebles, espacios especializados, instalaciones,...

A esta categoria y a esta clase de construcción responden esos "compactos" que, en principio, fueron sólo núcleos que formaban parte de esquemas de distribu. ción (años 40); posteriormente se convirtieron en "uto. pias autónomas" (años 60); luego en cápsulas técnicas (años 70); y, por último, han tomado cuerpo en los actuales "compactos" (años 80).

Al fin asistimos a la realidad de esos aparatos integra. dos, producidos industrialmente, flexibles, que generan y cualifican el espacio a su alrededor.

Sin embargo,... sin embargo, el soporte (todos los so. portes), justamente aquello que es lo más importante, aquello que es el fundamento sobre el que se ha de basar el desarrollo de la construcción, de la actividad industrial, de la vivienda de masas no responde aún, en la edificación convencional, a los planteamientos y necesidades que desde tales parámetros se plantean. Existen los materiales, existe la tecnología, existen las metodologias, existen los técnicos, existe la capacidad financiera, existe una demanda,... y, sin embargo,... sin embargo, qué sentido tiene todo ese estudio, toda esa capacidad, toda esa obcecación y persistencia en la búsqueda y rastreo de soluciones que vuelven una y otra vez a llamar con insistencia a la misma puerta.

¿Tienen razón de ser, o estarán equivocadas?

¿Se dan todas sus condiciones materiales, o son puro papel?, ¿por qué están ahi?, ¿por qué no se edifican?, ¿por qué sigue el arquitecto insistiendo?, ¿cuál es su papel en todo esto?, ¿dónde fallan los números?, ¿qué cultura es ésta?... 

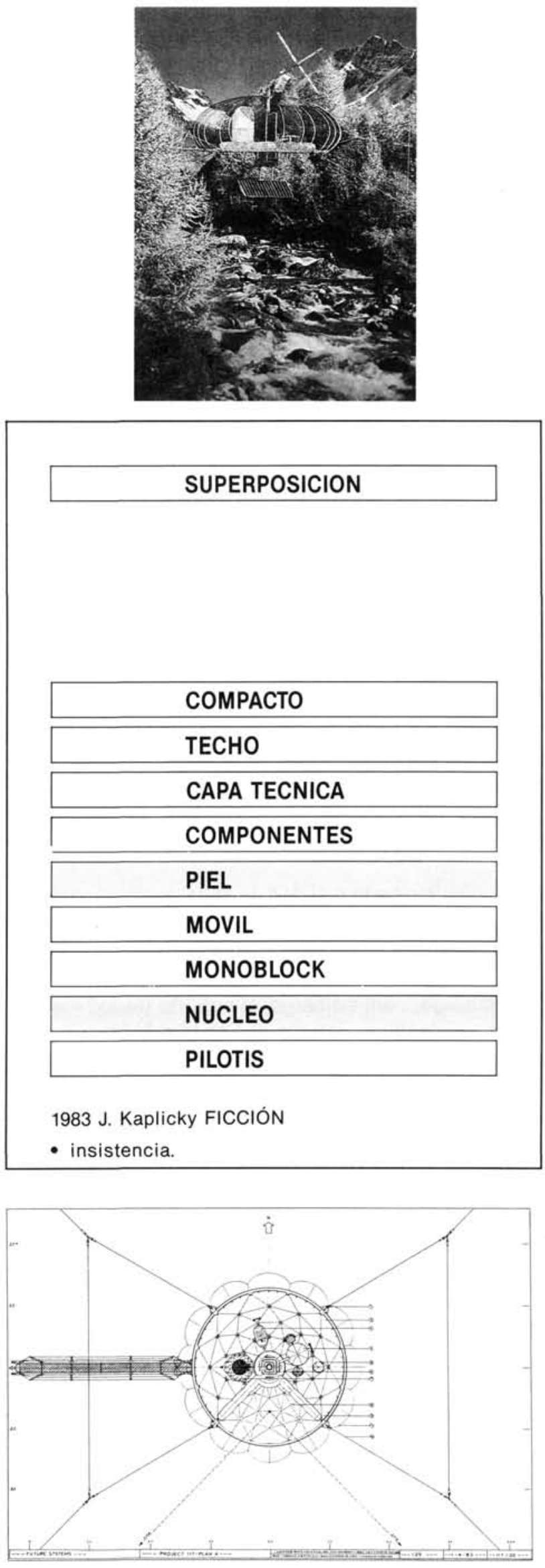

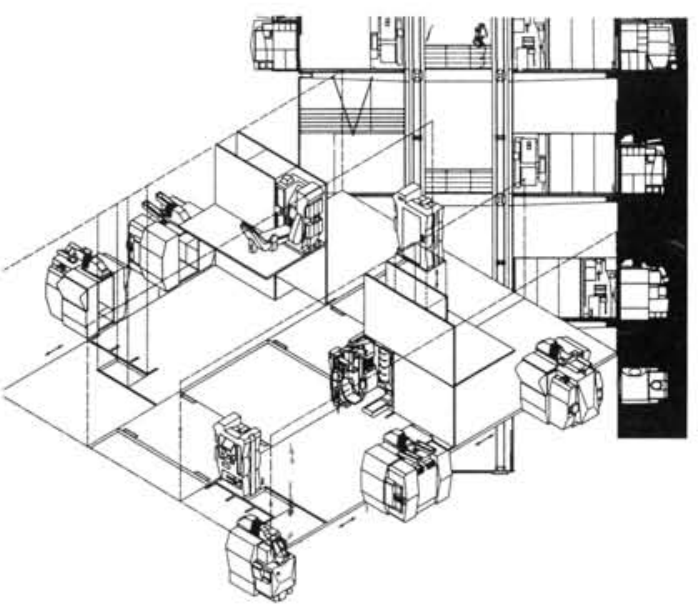

\begin{tabular}{|c|}
\hline INVERSION \\
\hline SUPERPOSICION \\
\hline СОМРАСТО \\
\hline TECHO \\
\hline CAPA TECNICA \\
\hline COMPONENTES \\
\hline PIEL \\
\hline MOVIL \\
\hline MOHBLOCK \\
\hline NUCLEO \\
\hline PILOTIS \\
\hline $\begin{array}{l}198911^{\text {er }} \text { Premio Europan en Austria } \\
\text { LOS URBANAUTAS }\end{array}$ \\
\hline $\begin{array}{l}\text { - se invierten los lugares de núcleo y comunica- } \\
\text { ciones (interiorlexterior), } \\
\text { - se libera al bloque de instalaciones de la ilumi- } \\
\text { nación y ventilación artificial. }\end{array}$ \\
\hline
\end{tabular}

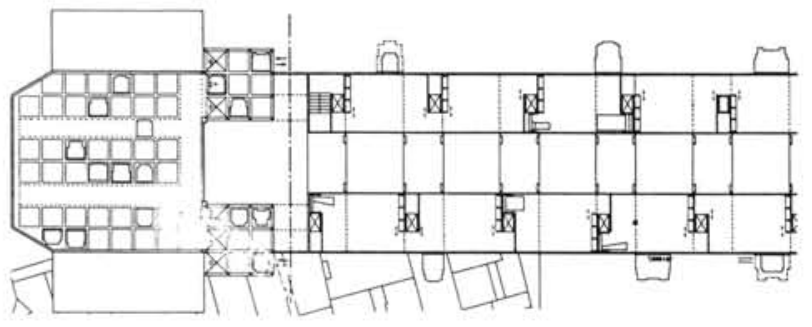



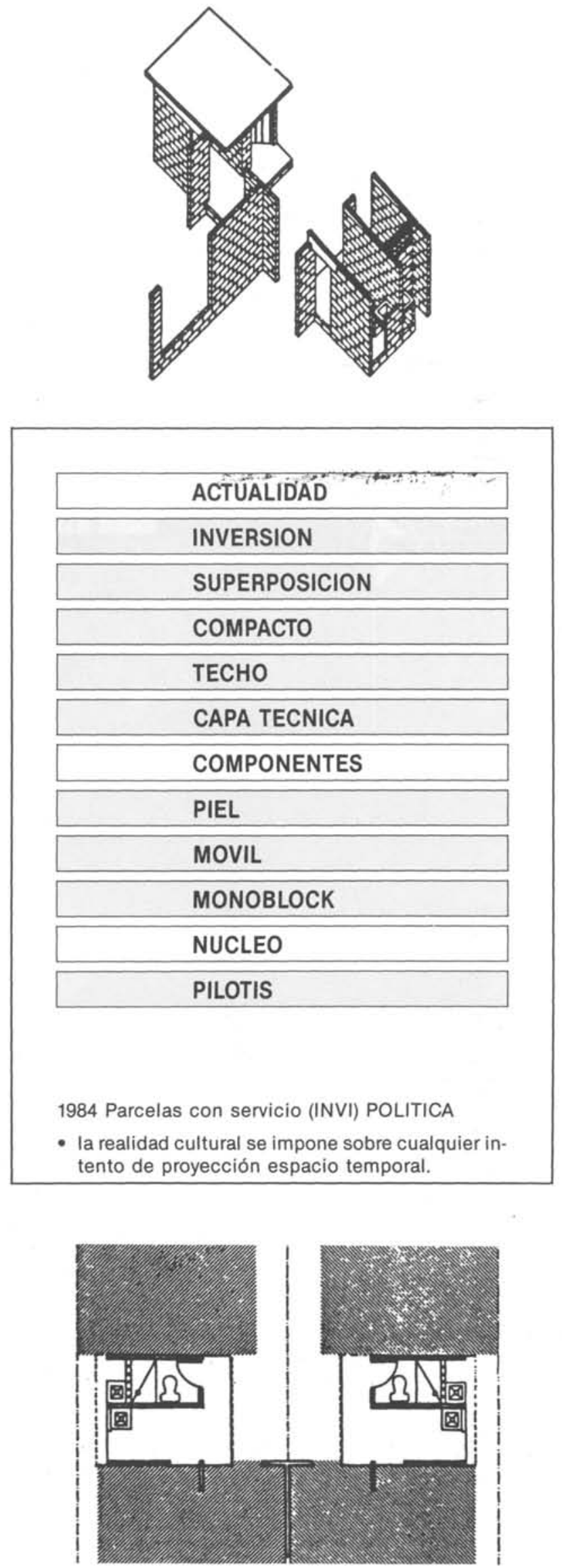

Pero los "compactos" están ahi; son aparatos producidos industrialmente que influyen en las condiciones de habitabilidad y construcción de la vivienda; ¿llegarán a encontrar su lugar en la misma, o tendremos que camuflarlos con cualquier carcasa plástica que nos recuerde algún capitel repleto de "hojarasca"?

Mientras, ellos vienen a decirnos:

la evolución de la tecnología de las instalaciones domésticas influye de mane. ra constante en la evolución de la vivien. da.

Nosotros, podremos escucharlo o no, creerlo o no, seguir parados o no: los huéspedes están servidos.

\section{Mesa de trabajo para el Lloyd's Center (R. Rogers).}

En este proyecto la investigación se enfocó hacia el desarrollo del control local a base de elementos adaptables que integrasen las funciones tradicionales en un despacho con un control individual del medio.

El sistema está basado en un módulo de trabajo integral y móvil. Aunque está concebido para conformar toda una oficina, puede usarse también como unidad separada.

El ensamble de estos componentes estándar permite, en función de la pericia del usuario, infinidad de combinaciones.

El módulo se mueve y apoya sobre una delgada capa de suelo técnico, y su conexión con el mismo le facilita: suministro eléctrico, recepción de datos y tuberias de fluido.

El módulo se construye partiendo de los siguientes componentes básicos:

- Chasis. Consiste en un mástil vertical que suministra aire, corriente eléctrica y línea informática; se desplaza sobre una armadura con ruedas.

- Módulo de control. En él se encuentran los elementos de enchufe al mástil para los diferentes servicios. Distintos componentes mecánicos y electrónicos pueden ser conectados al módulo. Este módulo de control es manejado a través de un teclado y una pantalla. A través de esta unidad el usuario activa y desactiva las funciones del módulo. Un carril de corriente y un sistema de adaptadores permite enchufar el equipo de iluminación, etc. 


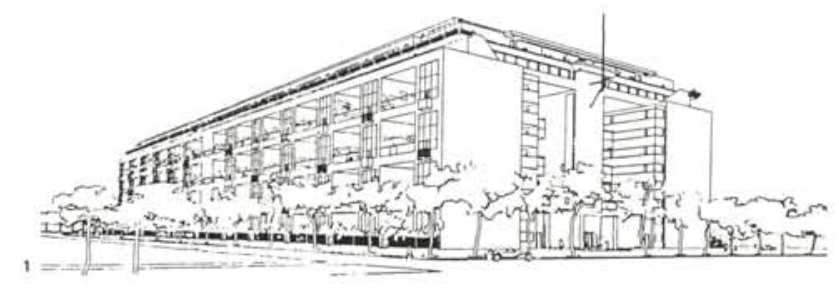

ZIP.UP N. 1

Richard Mas Rogers

1968

Inmuebles Villa

Le Corbusier

1925 Pabellón "Espirit-Nouveau"
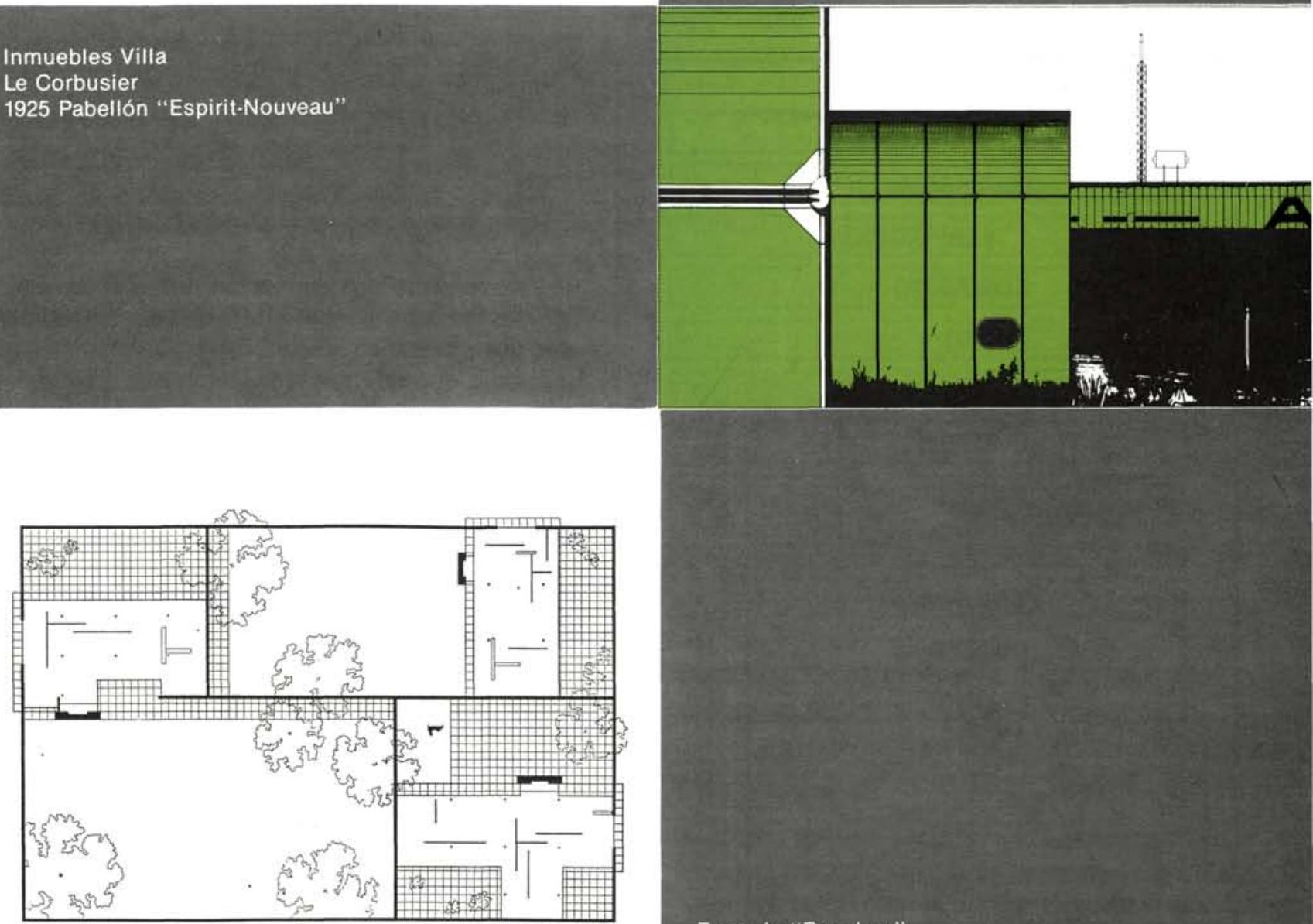

Zona de "Ranchos"

Autoconstrucción en Caracas

1984

Casa Patio

Mies V. der Rhoe

1931

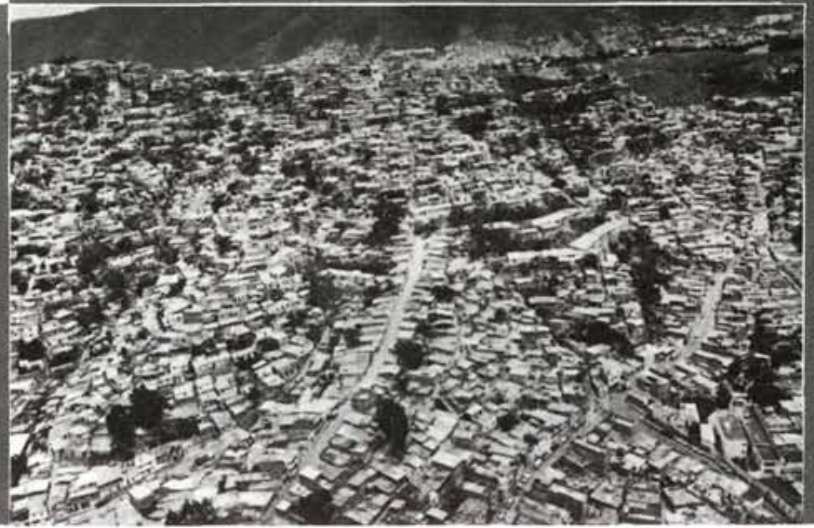


- Palio ambiental. Se engancha en la parte superior del mástil sobre la superficie de trabajo, como si fuera un paraguas. Produce aislamiento acústico (absorción), iluminación (mediante un plafón), amortiguación del sonido interior y difusor de aire. El usuario puede controlar su altura y posición, asi como regular la luz, aire y funciones acústicas.

- Tablero de trabajo. Este se pliega sobre el mástil principal cuando no esté en uso, o cuando se quiera mover el módulo. Otros mecanismos permiten ajustar la altura de la misma. El tablero está equipado con un carril de suministro eléctrico e informático.

- Plataformas para máquinas. Los teclados de máquinas de escribir, procesadores, etc., pueden ser utilizados cómodamente a la vez mediante un sistema de bateas pivotantes. Un flexo puede ser también enganchado al mástil principal para suministrar iluminación local sobre esta ocupación.

- Pantalla acústica. Una pantalla acústica plegada permite definir un recinto cerrado para reuniones o realizar algún trabajo privado.

\section{Ergo (Carlo Urbinati).}

El programa de ERGO encuentra en el cuerpo situado sobre la base del lavabo su máximo punto de interés.

A partir de los usos actuales del cuarto de baño, un número de servicios y varias clases de equipamiento es. tán concentrados en este elemento:

- Sistema de iluminación;

- una fuente graduada de aire caliente;

- reloj digital;

- una radio $\mathrm{AM}-\mathrm{FM}$;

- termómetro de ambiente;

- calentador de toallas;

- espejo iluminado en todas direcciones;

- suministrador de jabón líquido;

- repisas para objetos.

Todo ello combinado en una unidad aislada, fabricada en un material antichoque y de alta resistencia.

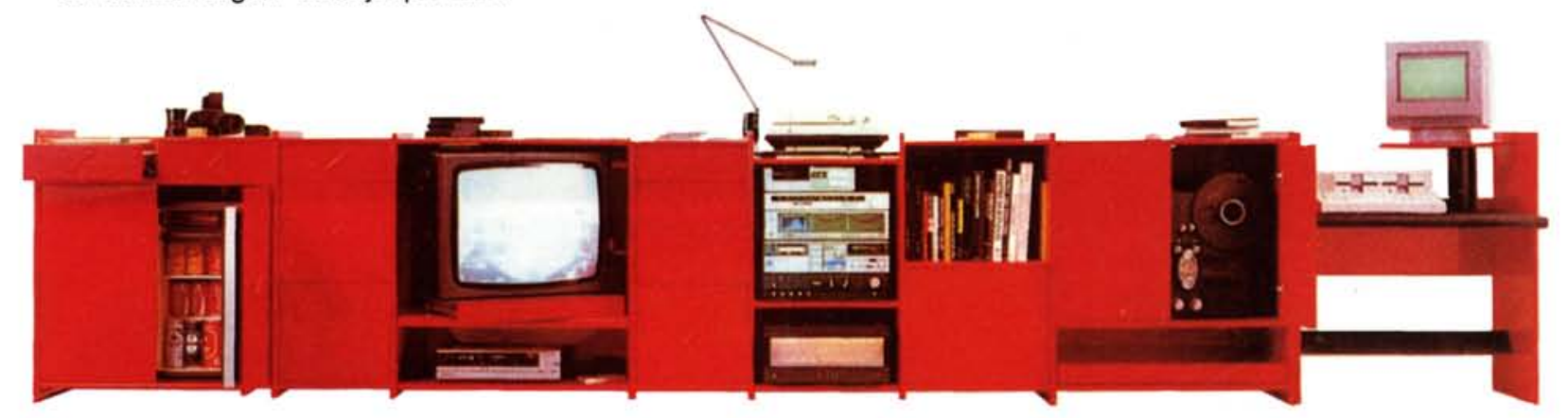

\section{Pragma (Giovani Offredi).}

El proyecto desarrolla, de forma especial, la definición de una zona de trabajo que está organizada a partir de las superficies de apoyo, y sus elementos de fijación están destinados a actuar como contenedores.

La zona de trabajo se caracteriza sobre todo por una serie de contenedores, con una altura de $48 \mathrm{~cm}$, una profundidad de $33 \mathrm{~cm}$ y sujetos en su parte inferior por los elementos de fijación. Éstos actúan como entrepaños cuando están abiertos y se cierran como puertas horizontales de dos hojas.

Otra particularidad del modelo son los aparatos eléctricos. Rompiendo con el resto de los otros elementos, éstos se evidencian y remarcan mediante un tratamiento frontal de sus superficies en acero inoxidable.

\section{Acordo (Giorgo Salvini).}

Dispone de componentes modulares sobre los cuales se pueden colocar sistemas audiovisuales. Lacados en su superficie, los módulos están diseñados para responder a las diferentes necesidades de aquellos que aman la música, la fotografía, son aficionados a las grabaciones de vídeo y a los computadores personales, etc. Entre los muchos accesorios incluidos son de destacar:

- El sitio para los amplificadores.

- El diseño para eliminar las reverberaciones acústicas.

- Hojas orientables para conseguir el estéreo; secciones intercambiables conformadas para almacenar discos; cassettes; videocassettes; compactdisc; etcétera.

- Una plataforma orientable para la TV. y otra especial para el P.C. 